\title{
POSIBILIDADES ESTRUCTURALES DE MATERIALES A BASE DE CAL COMO CONGLOMERANTE: PRIMEROS RESULTADOS
}

\author{
(STRUCTURAL POSSIBILITIES FROM LIME AS BINDING MATERIAL: FIRST RESULTS)
}

Enrique Estrada y José Miguel Barbero, Arquitectos

Equipo Viviendas de Muy Bajo Coste, IETcc - CSIC

\section{RESUMEN}

La cal es un material abundante y cuya producción suma a una tecnología muy accesible la ventaja de poder materializarse en pequeñas plantas dispersas, para poder reducir al mínimo la componente de costo debida al transporte.

En el presente trabajo se ha planteado la posibilidad de obtener hormigones con fines estructurales, para viviendas de baja altura y que sean de fácil obtención, a base de cal y cenizas volantes o cenizas de cáscara de arroz, variando sus proporciones, grados de finura y estudiando la infuencia de distintos factores en la resistencia inicial y final.

Se han obtenido buenos resultados, principalmente con ceniza de cáscara de arroz y cal con resistencias a 60 días superiores a los $15 \mathrm{MPa}$.

\section{INTRODUCCION}

Este trabajo se centra en encontrar distintas alternativas para la utilización de hormigones a base de cal (cal más adiciones) que pudieran utilizarse con fines estructurales en viviendas de baja altura.

Ante la cuestión de por qué hormigones a base de cal, cuando la diferencia de costos cal/cemento no es sustancial y la primera presenta desventajas claras, en cuanto a resistencia inicial, hay que tener en cuenta que el presente trabajo se realiza en el marco del Proyecto "Materiales, Tecnologias y Prototipos de Viviendas de Muy Bajo Coste" y que sus resultados pueden ser aplicables en paises en vias de desarrollo en los que el precio de un saco de cemento importado puede llegar a multiplicarse por diez respecto al precio en su pais de origen, cambiando el equilibrio antes aludido entre cemento y cal. Por otra parte, la tecnologia necesaria para asumir la producción de cemento es alta y exige inversiones importantes (aproximadamente 1.000 .000 de $\$$ para una producción de 10 t/dia) (1) para la realización de grandes plantas cementeras que, centralizando la producción, hacen que el factor transporte tome un protagonismo desproporcionado en paises con poca o nula infraestructura viaria.

En contrapartida, la producción de cal requiere una tecnologia muy apropiada a la que a una pequeña inversión, habria que añadir la ventaja de poder materializarse en pequeñas plantas dispersas; con lo que la componente de costos de transporte, se puede reducir al minimo.

Con estos objetivos se abordó este trabajo experimental destinado a conseguir hormigones a base de cal y subproductos industriales 0 agricolas. Una primera dificultad fue la ausencia de normativas especificas para este tipo de hormigones, adoptando como referencias más próximas al tema tratado, las Normas siguientes:

\section{- ASTM C821-74; ASTM C141-67 (R78); ASTM C49-57 (R79); UNE 7189-62.}

Todas ellas se refieren a morteros y no a hormigones, por lo que han tenido un valor de referencia y orientación, modificándose algunos puntos, a los que se hará referencia. 


\section{OBJETIVOS}

Este trabajo se centra en la investigación de las posibles caracteristicas estructurales de hormigones a base de cal como aglomerante, añadiéndole según el caso: ceniza volante en distintas proporciones y en tres grados de finura; ceniza de cáscara de arroz en distintas proporciones y en dos grados de finura; comparando los resultados de resistencia a compresión a 7,28 (ASTM C141-67) y 60 dias, con dosificaciones patrón, conteniendo un $100 \%$ de cal como aglomerante.

Se estudian: la influencia del factor agua/conglomerante $(\mathrm{a} / \mathrm{c})$ en la resistencia de las probetas; la influencia del \% de aglomerante, respecto al árido y el \% de adición, respecto al aglomerante.

\section{MATERIALES Y METODOS}

\subsection{Materiales}

Se enumeran a continuación las principales caracteristicas de los materiales empleados en la elaboración de los hormigones realizados en este trabajo:

- Cenizas volantes procedentes de la central térmica de Soto de Ribera (Asturias) considerada como parte integrante del aglomerante.

- Cal apagada (hidróxido de cal) con un contenido de $\mathrm{Ca}\left(\mathrm{OH}_{2}\right)$ superior al $90 \%$ y de $\mathrm{CO}_{2}$ inferior al $7 \%$.

- Arena del Manzanares: tamaño máximo 9,11 $\mathrm{mm}$; módulo granulométrico 3,13 y peso específico $2,60 \mathrm{gr} / \mathrm{cm}^{3}$.

- Gravilla del Jarama: tamaño máximo 19,05 $\mathrm{mm}$; módulo granulométrico 7,55 y peso especifico $2,10 \mathrm{gr} / \mathrm{cm}^{3}$.

- Ceniza de cáscara de arroz (RHA): procedente del horno preparado al efecto por el equipo de investigación (2).

\subsection{Metodología}

Se han estudiado los siguientes parámetros:

- Evolución de la resistencia de todas las probetas en función del tiempo, rompiéndose a 7,28 y 60 dias y registrándose los valores en los correspondientes diagramas tensión/ tiempo para todas las dosificaciones.

- Influencia del contenido en agua $(a / c)$ en la resistencia. Realizándose todas las dosificaciones para tres relaciones a/c de $0,7,0,6$ y 0,5 , no bajándose de 0,5 por problemas de trabajabilidad y no superando el valor 0,7 por mermas excesivas en la resistencia.

- Influencia de la finura y de la cantidad de adición en la resistencia final. Las adiciones se han utilizado en la siguiente forma:

- Ceniza volante de Soto de Ribera en tres finuras: natural $\left(2.954 \mathrm{~cm}^{2} / \mathrm{gr}\right.$ Blaine), y triturada con molino de bolas hasta alcanzar las finuras de: 4.500 y 8.000 $\mathrm{cm}^{2} / \mathrm{gr}$ Blaine.

- Ceniza de cáscara de arroz quemada a 985 $\pm 100 \mathrm{C}(2)$.

Tanto las cenizas volantes, como las cenizas de cáscara de arroz, se han mezclado con cal apagada, en los siguientes porcentajes:

- $0 \%$ de ceniza volante $+100 \%$ de cal (patrón).

- $20 \%$ de ceniza volante $+80 \%$ de cal (cenizas en las tres finuras).

- $30 \%$ de ceniza volante $+70 \%$ de cal (cenizas en las tres finuras).

- $40 \%$ de ceniza volante $+60 \%$ de cal (cenizas en las tres finuras).

- 0\% de RHA + $100 \%$ de cal (patrón).

- $30 \%$ de RHA $+70 \%$ de cal (RHA en las dos finuras).

- $55 \%$ de RHA $+45 \%$ de cal (RHA en las dos finuras).

- $70 \%$ de RHA + $30 \%$ de cal (RHA en las dos finuras).

Las proporciones de adición no se mantuvieron constantes persiguiendo obtener las mayores resistencias, dado que existe bibliografia abundante, en la que se aconsejan proporciones más altas de RHA (3).

- Influencia de la proporción de aglomerante (cal + adición) respecto al árido, fijando tres valores del 15,25 y $35 \%$, respectivamente de conglomerante respecto al árido.

- Influencia sobre dosificaciones seleccionadas en el proceso anterior de pequeñas adiciones de yeso o cemento. En su resistencia a compresión.

En el esquema de la Fig. 1 se recogen de forma sistematizada las composiciones de las distintas dosificaciones, asignándole a cada una de ellas un número de clave del 1 al 54 , al 
Informes de la Construcción, Vol. 38, n.o 385, noviembre, 1986

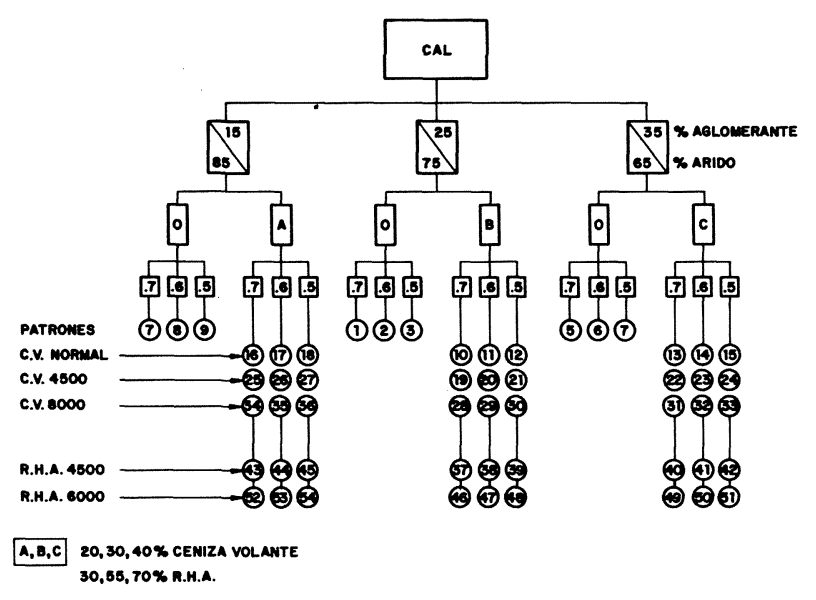

Fig. 1

que se referirán los resultados a lo largo del trabajo. La mencionada figura 1 trata de reflejar de forma gráfica la metodología seguida en la realización del trabajo.

\subsection{Confección}

Para la realización de las probetas, curado y rotura, se han seguido las orientaciones de la Norma ASTM C141-67 (Hydraulic hydrated lime for structural pourposes) con la salvedad de que los cubos se han realizado de $7 \times 7 \times 7 \mathrm{~cm}$, en lugar de $5 \times 5 \times 5$ (UNE 7/189/62), por tratarse de hormigones, en lugar de morteros.

Las roturas se han realizado a 7,28 y 60 dias, a compresión simple con prensas tipo Amsler 50 D 73, de $50 \mathrm{Mp}$ de capacidad máxima para ensayos a compresión; y prensa tipo Toni-Technik, de $20 \mathrm{Mp}$ automática, para ensayos a compresión, con una dispersión entre ambas menor de $10 \%$.

\section{EVOLUCION DE LA RESISTENCIA DE LAS PROBETAS, EN FUNCION DEL TIEMPO}

La Fig. 2 recoge los resultados de los ensayos a compresión de las 54 dosificaciones estudiadas. En los seis esquemas que constituyen la Fig. 2, se ha seguido la misma sistemática: sobre unas coordenadas cartesianas, en ordenadas se han registrado resistencias a compresión -valores medios de las roturas, expresados en MPa. En abscisas se han situado las 54 dosificaciones, representando para cada una de ellas los resultados mediante tres barras

correspondientes a los resultados a 7, 28 y 60 dias, respectivamente, colocados en este orden de izquierda a derecha en todos los casos, y en la misma posición relativa que en la Fig. 1 .

En los cinco esquemas de la Fig. 3, se recogen igualmente, resultados a compresión en MPa de distintas dosificaciones, representándose en abscisas dos tipos de factores: relaciones a/c y proporción de conglomerante.

Los valores indicados mediante barras negrasvalores puramente orientativos-, son las medias de los correspondientes para el 15,25 , y $35 \%$ de conglomerante.

Para la ceniza de R.H.A. con $a / c=0,5$ y $15 \%$ de conglomerante, las roturas a compresión presentan valores extremadamente bajos, del orden de $2 \mathrm{MPa}$, creciendo significativamente al pasar al $25 \%$ y $35 \%$ de conglomerante.

Este comportamiento parece compatible con el hecho de que la RHA necesita más agua para su trabajabilidad.

Como era de esperar a semejanza de los hormigones convencionales, la resistencia final decrece con el aumento del agua de amasado, siendo las cenizas volantes mucho más sensibles a esta variable, ya que se observan variaciones del $100 \%$ de la resistencia para incrementos de dos puntos en la relación a/c frente a variaciones del $27 \%$ en el caso de RHA.

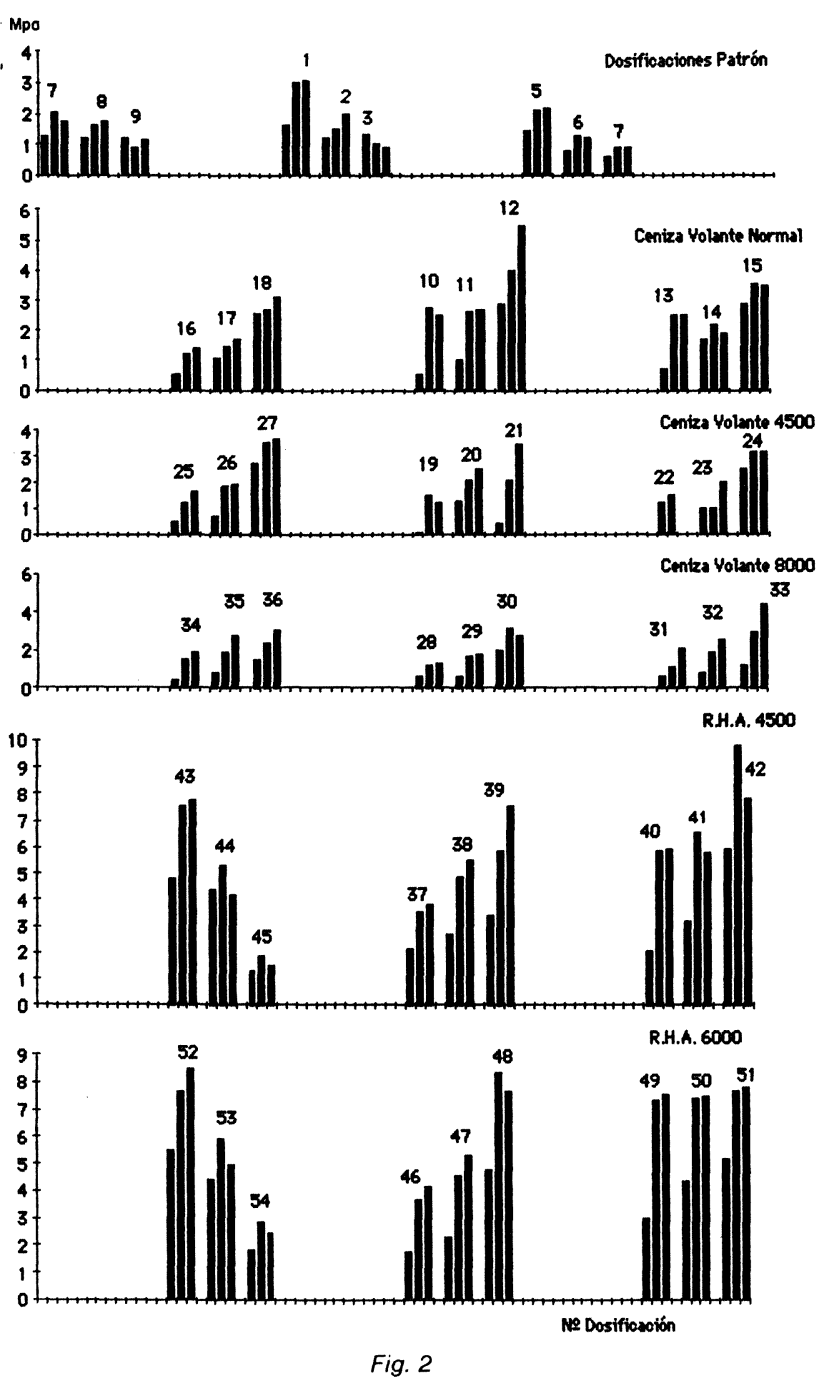



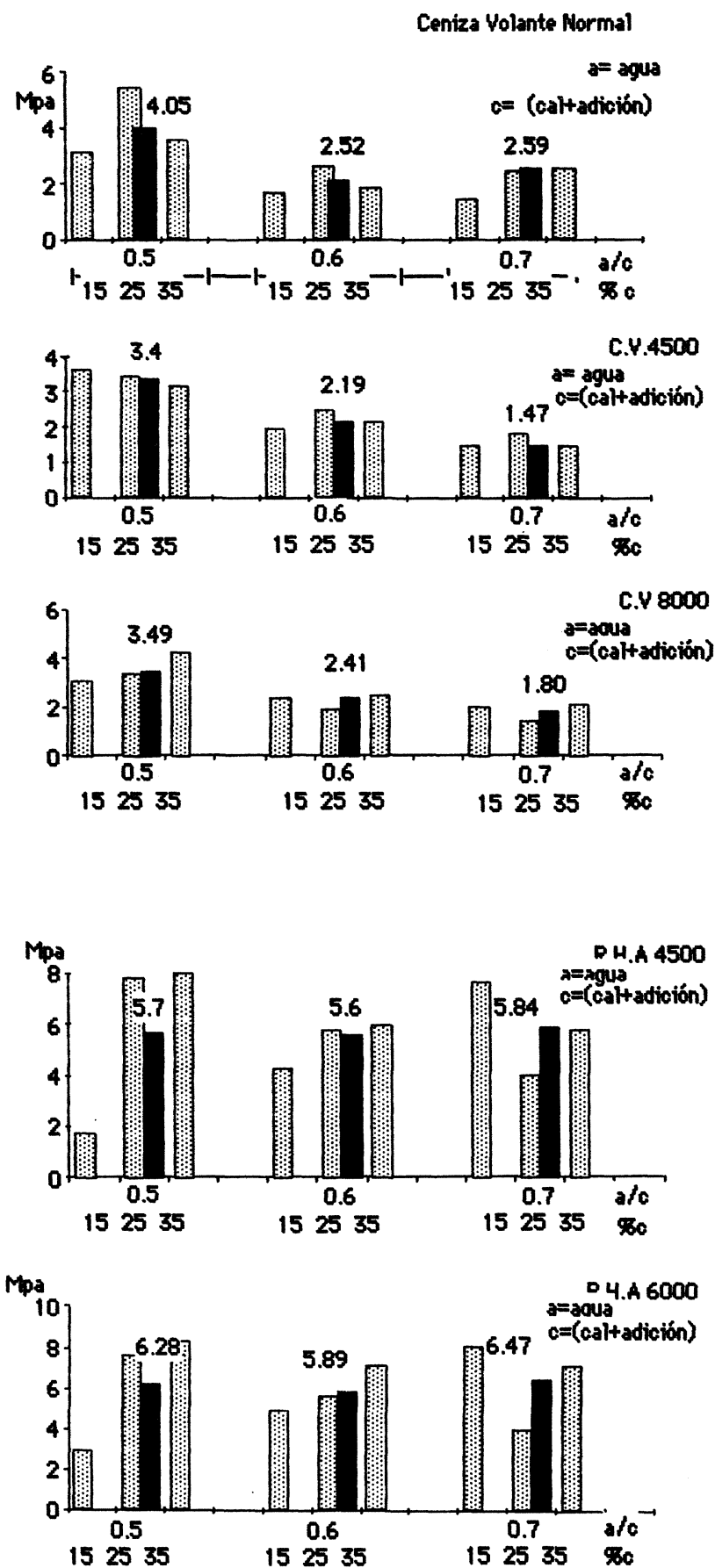

Fig. 3. - Influencia de la relación a/c en la resistencia final.

\section{INFLUENCIA DEL GRADO DE FINURA EN LA RESISTENCIA FINAL}

Existe abundante literatura en la que se acepta que el grado de puzolanicidad es proporcional, en gran parte, al grado de finura o molienda de las cenizas (4) (5). Observando la Fig. 4 en la que se representan valores medios, parece constatarse este hecho para el caso de la RHA; pero no ocurre lo mismo para el caso de las cenizas volantes. Puede observarse que, aún en el caso de las cenizas, RHA, las diferencias no

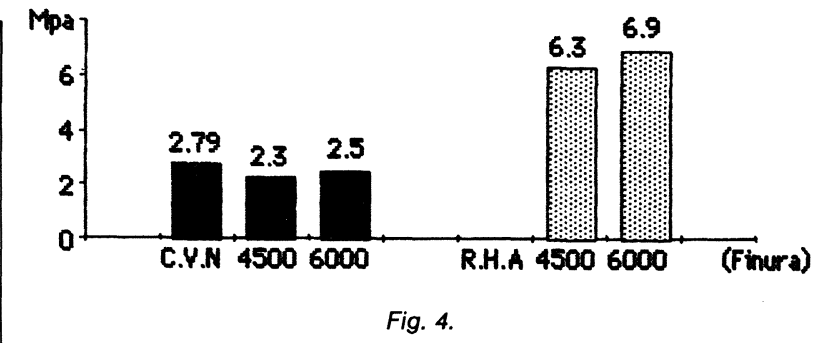

son muy significativas (menores del 10\%), por lo que habria que plantearse la conveniencia o no del mayor consumo de energia necesario en el molido para tan escaso incremento de la resistencia conseguida.

\section{INFLUENCIA DE LA PROPORCION DE CONGLOMERANTE EN LA RESISTENCIA}

En los, cinco esquemas de la Fig. 5, se recogen los resultados correspondientes a las cenizas volantes y al RHA.

En cenizas volantes no aparece clara la relación creciente, entre el contenido de conglomerante
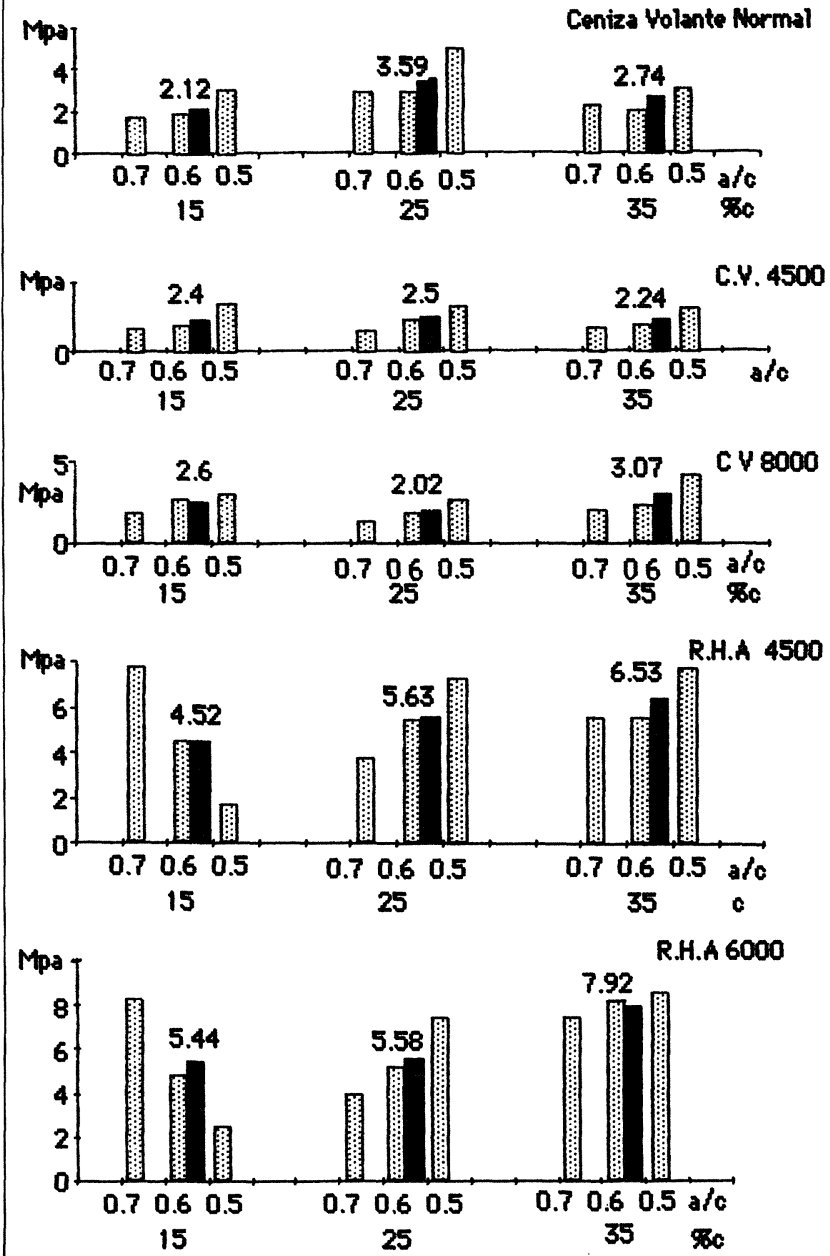

Fig. 5. - Influencia del tanto \% de conglomerante en la resistencia final. 
y la resistencia final. Los mejores resultados se presentan para un contenido del $25 \%$, aunque hay excepciones en función del grado de finura, ya que para un $35 \%$ de CV 8000 se dan los mejores resultados.

Empleando RHA, la relación conglomerante resistencia aparece mucho más clara, el crecimiento es casi lineal, produciéndose los mejores resultados para relaciones del $35 \%$ del conglomerante, que fue el máximo ensayado, lo que recomienda probar con proporciones mayores en posteriores trabajos.

Merece la pena comentar la circunstancia que se detecta al utilizar RHA, ya que la resistencia de las probetas con un $15 \%$ de conglomerante presenta valores decrecientes conforme se pasa de relaciones a/c de 0,7 a 0,6 y de este valor a 0,5

\section{INFLUENCIA DE LA PROPORCION DE ADICION FRENTE AL CONGLOMERANTE CAL EN LA RESISTENCIA FINAL}

A la vista de los esquemas de la Fig. 6 puede deducirse la dificultad existente en obtener conclusiones generales dada la gran diversidad de comportamientos detectados y que, en el caso de las puzolanas, dependen de infinidad de variables: "Las propiedades técnicas del producto varian según la composición de las materias primas; la puzolana y la cal, que varian en cada caso y yacimiento y dependen también del procesamiento. Influye la correcta cochura y apagado de la cal. Otra variante es la granulometria, o sea, el tipo de molienda y clasificación de las partículas" (6).

Mpo
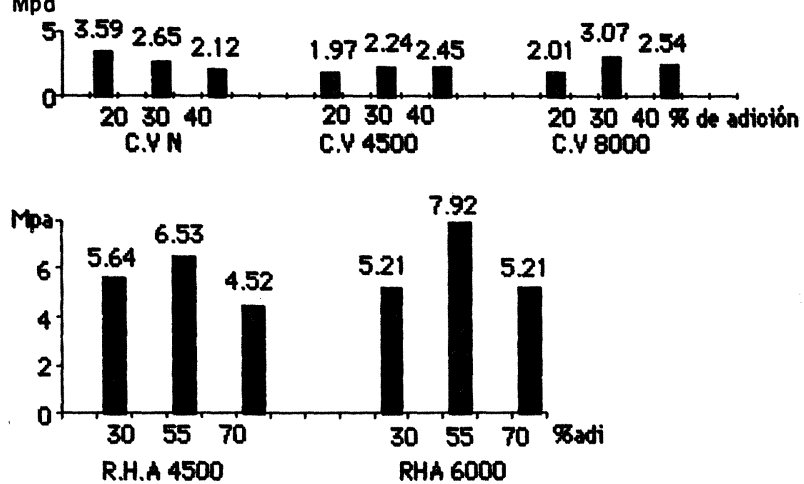

Fig. 6. - Influencia de la proporción de adición frente al conglomerante cal en la resistencia final.

\section{SELECCION DE LAS DOSIFICACIONES MAS IDONEAS}

En función de los resultados obtenidos para valores medios de todas las dosificaciones y atendiendo a los parámetros anteriormente citados, se obtendrian los perfiles óptimos teóricos, para el caso de las cenizas volantes y la RHA, que serian los siguientes:

\begin{tabular}{|l|c|c|c|c|c|}
\hline & a/c & Finura & $\% \mathrm{c}$ & $\%$ adición & Dosif. n.o \\
\cline { 2 - 6 } $\begin{array}{l}\text { CENIZA VOLANTE } \\
\text { CENIZA DE CASCARA } \\
\text { DE ARROZ }\end{array}$ & 0,5 & Normal & 25 & 20 & 12 \\
\hline
\end{tabular}

En la figura 7 se recoge la evolución de resistencias a 7,28 y 60 dias para las dosificaciones seleccionadas, así como para la patrón de cal.

Referenciando las dosificaciones seleccionadas a su correspondiente patrón de cal sin adiciones, se consiguen incrementos en la resistencia inicial del $75 \%$ para cenizas volantes y del $215 \%$ para la RHA, así como incrementos del 82 y $188 \%$ respectivamente, en su resistencia a 60 dias. Esto es interesante, si se tiene en cuenta que uno de los principales inconvenientes de la cal como conglomerante, es su baja resistencia inicial.

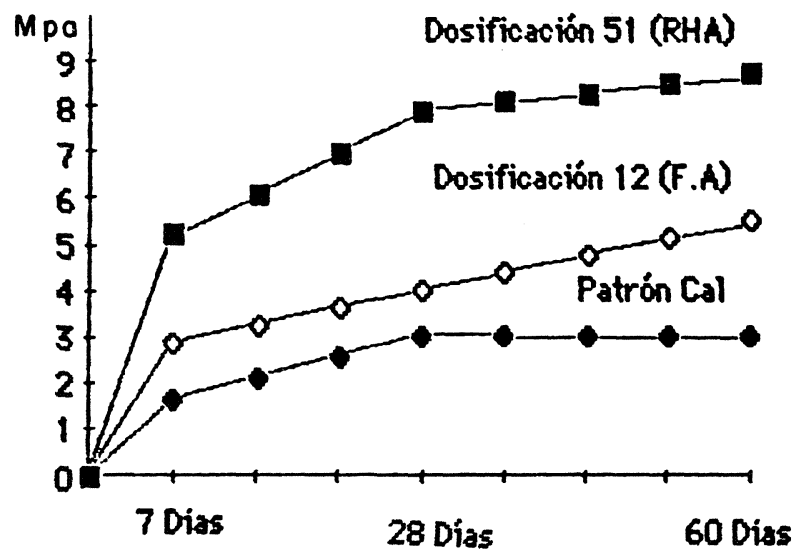

Fig. 7. - Diafragma tensión/tiempo de las dosificaciones óptimas.

\section{INFLUENCIA DE PEQUEÑAS ADICIONES DE YESO Y CEMENTO EN LAS DOSIFICACIONES SELECCIONADAS}

A partir de las dosificaciones seleccionadas, la número 12 para las cenizas volantes y la número 51 para la RHA, se ha estudiado la influencia en la resistencia a compresión de las probetas al sustituir pequeñas proporciones de cal por yeso o cemento, en distintos \% del 3,6 y 9 para el yeso, y del 8,16 y 24 para el caso del cemento.

La Fig. 8 muestra claramente el negativo efecto sobre la resistencia a la compresión de la adición de yeso en la dosificación 12. La caida 


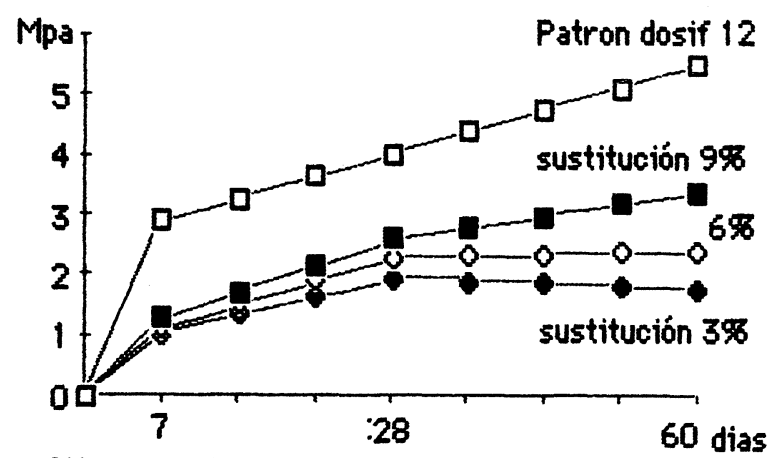

Inf Yeso en C.Y

Fig. 8. - Influencia del yeso sobre ceniza volante.

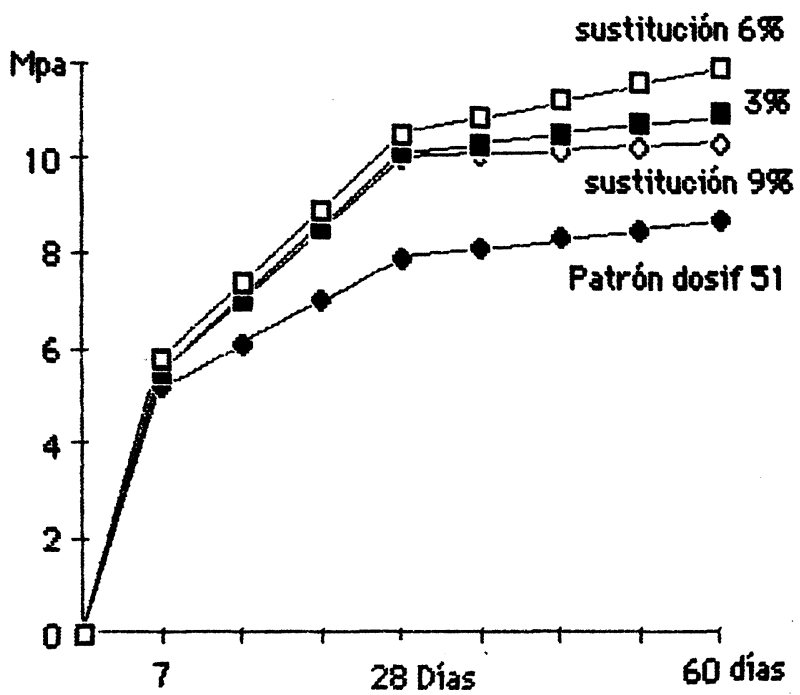

Inf yeso en R.H.A

Fig. 9. - Influencia del yeso en R.H.A.

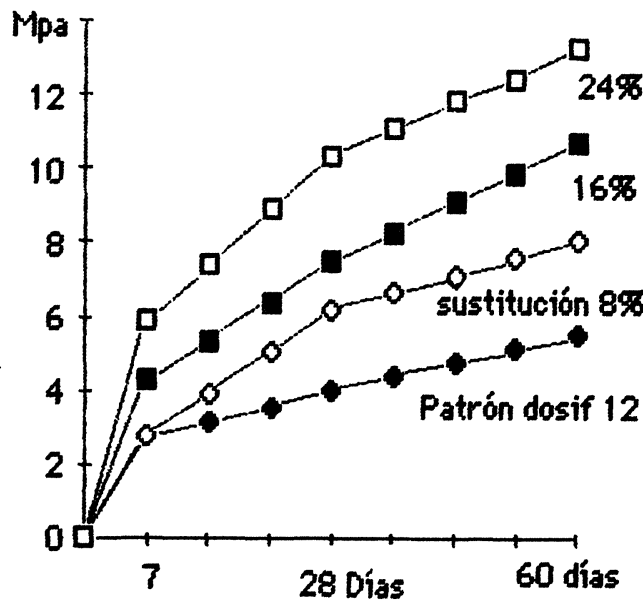

Inf cemento en C.Y

Fig. 10. - Influencia del cemento en Cenizas Volantes.

de resistencias a 7,30 y 60 dias, ocasionada por la sustitución de un $3 \%$ de conglomerante por yeso, se mejora relativamente al pasar al $6 \%$ y de este valor al $9 \%$, lo que apunta hacia la consecución de mayores resistencias para mayores cantidades sustituidas.

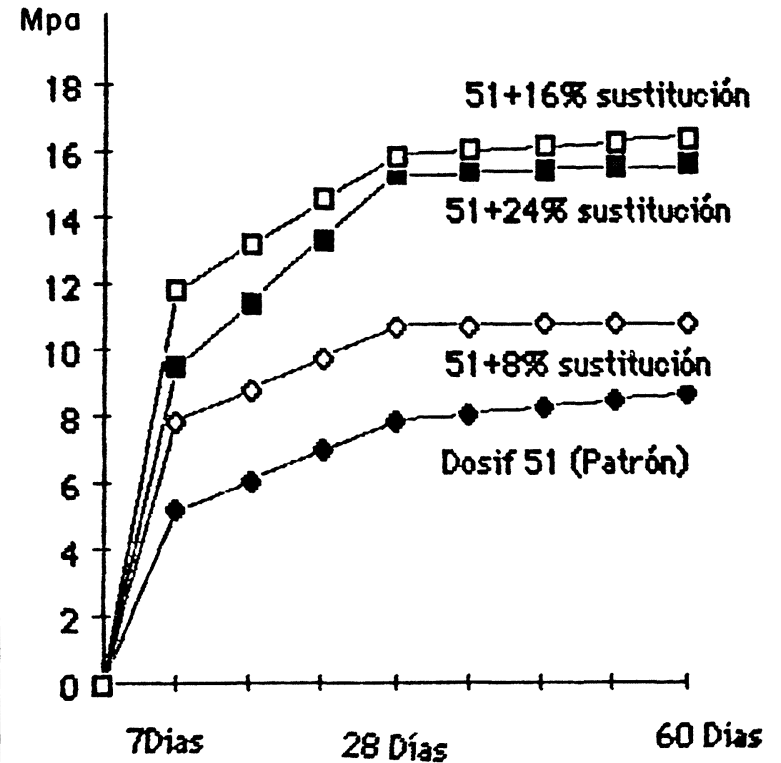

Fig. 11. - Influencia del cemento en ceniza de cáscara de arroz.

El efecto es bien distinto en la dosificación 51 a base de ceniza de cáscara de arroz. A todas las edades las resistencias aumentan para sustituciones del $3 \%, 6 \%$ y $9 \%$, presentando para este último porcentaje una caida relativa de resistencia, lo que induce a suponer, a falta de un trabajo más minucioso, que la cantidad de conglomerante sustituible óptima estará entre $3 \%$ y $6 \%$.

En las Figuras 10 y 11 se refleja, como era previsible, la beneficiosa influencia de la sustitución de conglomerante por cemento. En el caso de la dosificación patrón 12, los resultados son claros, no asi, en el caso de la dosificación 51 , a base de ceniza RHA, que muestra una inversión de la tendencia creciente al pasar del $16 \%$ al $24 \%$.

\section{CONCLUSIONES DIRECTAS}

Del conjunto de dosificaciones estudiadas, se pueden deducir una serie de conclusiones inmediatas sobre los aspectos estudiados:

a) Aparentemente, resulta viable desde el punto de vista estructural la utilización de este tipo de hormigones en viviendas de baja altura. Los resultados obtenidos no permiten juzgar aspectos: como adherencia, corrosión de armaduras, etc.

b) Un hormigón pobre de cemento Portland, supone aproximadamente un $12 \%$ de cemento respecto al peso total, para una resistencia de unos $21 \mathrm{MPa}$, la dosificación 51 con un $16 \%$ de sustitución de cal por cemento, equivale a un $2 \%$ de cemento respecto al peso total, para una resistencia 
aproximada de $16 \mathrm{MPa}$. Esto supone un consumo 6 veces menor de cemento para una pérdida de $28 \%$ de resistencia.

Por su parte la dosificación 12 con un $24 \%$ de sustitución de cal por cemento, presenta un $5,3 \%$ de cemento respecto al peso total, para una resistencia aproximada de 13 $\mathrm{MPa}$. Esto supone un consumo de cemento sólo 2,26 veces menor para una pérdida del $37 \%$ de resistencia.

c) Hormigones a base de cal ( $45 \%$ ) y ceniza de RHA (55\%), sin otro tipo de adición, proporcionan resistencias a 7 dias del orden de los $5 \mathrm{MPa}$ valor aceptable para edificaciones de poca altura, que son las que nos ocupan.

Si se adiciona un $16 \%$ de cemento respecto a la cal, es decir, para una dosificación: $29 \%$ de cal, $16 \%$ de cemento y $55 \%$ de RHA, los valores iniciales alcanzan aproximadamente los $12 \mathrm{MPa}$.

d) Las sustituciones por yeso perjudican en el caso de la cenizas volantes (pérdidas del $46 \%$ en resistencia) mientras que favorecen a la RHA (incrementos del $27 \%$ en resistencia).

e) Las sustituciones por cemento favorecen tanto a las cenizas volantes (incrementos del $94 \%$ en resistencia) como a las RHA (incrementos del $64 \%$ en resistencia).

f) El grado de molienda estará más condicionado por la energía consumida y su costo, que por las mejoras de resistencias obtenidas.

g) La variable que se ha mostrado más critica respecto a la resistencia obtenida, ha sido, en todos los casos, la relación agua/aglomerante.

h) La ceniza RHA ha dado mejores resultados absolutos que las cenizas volantes, se muestra menos sensible a variaciones en los parámetros estudiados y ofrece un comportamiento más uniforme, por lo que se muestra como un material muy adecuado para su utilización en países en vias de desarrollo de unas determinadas características.

\section{CITAS BIBLIOGRAFICAS}

(1) Primer simposio Nacional del Cemento. Cochabamba (Bolivia) 1981.
(2) J. SALAS, PERCY CASTILLO, M.a ISABEL SANCHEZ ROJAS, JANER VERAS: “Empleo de cenizas de cáscara de arroz como adiciones en Morteros". Materiales de Construcción n.o 203. IETcc. Julio/sept. 1986. ESPAÑA.

(3) RAY SMITH: «Rice Husk Ash Cement; Progress in development and aplicatión". Intermediate Technology Development Group Limited. Londres, 1984.

(4) UMBERTO COSTA, FRANCO MASSAZA: "Factors Afecting the with lime of italian Pozzolanas». Revista il Cemento n.०3, 1974

(5) LUIS ARMANDO ZURITA: "Contribución fisico-quimica al estudio de los materiales potencialmente puzolánicos». Centro de Investigaciones de Ingenieria. Guatemala C. A. Agosto, 1984

(6) E. PLOSKONKA: “Aprovechamiento de Materiales puzolánicos en Bolivia». No publicado. 1982, Bolivia.

\section{BIBLIOGRAFIA CONSULTADA}

- BIE KUL, SPENCE ROBIN: "Alternative Cements in India". Intermediate Technology Development Group Limited. 46 págs. Londres, 1976.

- UNCHS: «Building Lime». Building Digest n.o 8. Central Building Research Institute. Roorkee 1962.

- UMBERTO COSTA-FRANCO MASSAZA: «Fac tors affecting the reaction with lime of italian pozzolanas». Revista il Cemento n.o 3, 1974.

- LUIS ARMANDO ZURITA: "Contribución Fisicoquímica al estudio de los materiales potencialmente puzolánicos». Centro de Investigaciones de Ingenieria. Guatemala C. A. Agosto, 1984.

- E. PLOSKONKA: «Aprovechamiento de los Materiales Puzolánicos en Bolivia. No publicado, 1982.

- F. TRIVIÑO: «Puzolanas».

- UMBERTO COSTA, FRANCO MASSAZA: «Influence of the Thermal on the reactivity of some natural puzzolanas with lime». Revista il Cemento n.o 3, 1974.

- L. S. DENT GLASSER, N. KATAOKA: “On the dole of calcium in the alcali agregate reaction" University of Aberdeen 1981.

- ROBERT S. BOYNTON: “Chemistry and Technology of lime and limestone». Interscience Publishers.

- TANG BIHAO: «Research and aplication of largeescale precast. Wall-panels made with lime and flyash» CIB 83, pág. 265, 269.

- RAY SMITH: "Rice husk ash cement; progress in development and aplication". Intermediate Technology Development Group limited». Londres, 1984.

- J. SALAS, M. ALVAREZ, J. VERAS: “Lights/insulating concrete with Rice-Husk». Cement and Composites, Sheffield nk 1986. 